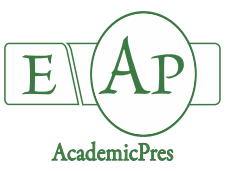

Sun W et al. (2021)

Notulae Botanicae Horti Agrobotanici Cluj-Napoca

Volume 49, Issue 3, Article number 12456

DOI: $10.15835 /$ nbha49312456

Review Article

\title{
The roles of a light-dependent protochlorophyllide oxidoreductase (LPOR), and ATP-dependent dark operative protochlorophyllide oxidoreductase (DPOR) in chlorophyll biosynthesis
}

\author{
Wenli SUN ${ }^{\text {1a* }}$, Mohamad H. SHAHRAJABIAN ${ }^{1 \mathrm{~b}}$, Qi CHENG ${ }^{2}$ \\ ${ }^{1}$ Chinese Academy of Agricultural Sciences, Biotechnology Research Institute, Beijing 100081, China; sunwenli@caas.cn \\ (*corresponding author); hesamshahrajabian@gmail.com \\ ${ }^{2}$ State Key Laboratory of North China Crop Improvement and Regulation, Hebei Agricultural University, College of Life Sciences, \\ Baoding, Hebei 071000, China; Global Alliance of HeBAU-CLS\&HeQiS for BioAl-Manufacturing, College of Life Sciences, Hebei \\ Agricultural University, Baoding, 071000,China; chengqi@caas.cn \\ ${ }^{a, b}$ These authors equally contributed to the work
}

\begin{abstract}
Chlorophyll is a green photosynthetic pigment, and photosynthesis drives the global carbon cycle. The reduction of protochlorophyllide (Pchlide) to chlorophyllide (Chlide) in the penultimate stage of biosynthesis of chlorophyll (Chl) is catalysed by light-independent protochlorophyllide reducatse (DPOR), and the lightdependent protochlorophyllide oxidoreductase (LPOR). The search was done to all manuscript sections according to terms chlorophyll, a light-dependent protochlorophyllide oxidoreductase, ATP-dependent dark operative protochlorophyllide oxidoreductase, chlorophyll, photosynthesis and chlorophyllide. Within the framework of photosynthesis and chlorophyll, this review article was aimed to provide an overview of the functional studies in chlorophyll biosynthesis, protein crystal structure, disclosure of action mechanisms, and possible future available direction of LPOR and DPOR in the biosynthesis of chlorophyll.
\end{abstract}

Keywords: chlorophyll; chlorophyllide; DPOR; LPOR; protochlorophyllide oxidoreductase

\section{Introduction}

\section{Chlorophyll and photosynthesis}

Chlorophyll is the main driving engine for photosynthesis (Hunter et al., 1994; Sun et al., 2019; Lu et al., 2020). It is the sole compartment containing green pigment where photosynthesis takes place within the cell (Humphrey, 1980; Mandal and Dutta, 2020; Sun et al., 2021). Chloroplast is one of the three kinds of plastids found only in plant cells (Mandal and Dutta, 2020). Chlorophyll has a tremendous capacity to trap light energy and apply in photolysis of water molecules to replenish the reducing power of the cells, which is necessary in carbon assimilation in subsequent steps of photosynthesis (van der Tol et al., 2009; Soleymani et al., 2016; Grajek et al., 2020; Mandal and Dutta, 2020; Shahrajabian et al., 2021).

Solar induced chlorophyll fluorescence (SIF) denotes reemitted light in the 650-850 nm range from the chlorophyll-a pigment, which is linked to initial steps in photosynthesis (Frankenberg and Berry, 2018). In some plants like peanut, Arachis hypogaea L. Golden2-like 1) (AhGLK1) activates the expression of AhPORA 
to promote chlorophyll biosynthesis, and that AhGLK1 physically interacts with Arachis hypogaea L. histone deacetylase 1) (Li et al., 2019; Liu et al., 2020). In photosynthesis, electrons are transported in the thylakoid from photosystem (PS) II to PSI and finally to $\mathrm{NADP}^{+}$in the storma, and electron transport results in the generation of the transthylakoid protein motive force that drives ATP synthesis (Gotoh et al., 2010). The main photosynthesis is $6 \mathrm{CO}_{2}+12 \mathrm{H}_{2} \mathrm{O} \rightarrow \mathrm{C}_{6} \mathrm{H}_{12} \mathrm{O}_{6}+6 \mathrm{H}_{2} \mathrm{O}+6 \mathrm{O}_{2}$. The photosynthesis process is also influenced by micronutrients such as boron, iron, manganese, copper and zinc through various modes of actions.

Chlorophyll $a(\mathrm{Chl} a)$ is the universal photosynthetic pigment which is available in most oxygenic photosynthetic organisms, and performs all of the above functions (Bjorn et al., 2009). Chl $b$ and other accessory pigments are mainly active in light-harvesting complexes (LHCs), and are expected to improve lightharvesting efficiency (Nick et al., 2013; Chen et al., 2014; Voltsekhovskaja and Tyutereva, 2015). Chlc is another accessory pigment involved in light harvesting and is found in heterokont algae descended from secondary endosymbiosis (Green, 2011). In $\mathrm{C}_{3}$ plants, the leaves do not have Kranz anatomy, Ribulose 1,5bisphosphate (RUBP) is the first acceptor of $\mathrm{CO}_{2}$, phosphoglycerate (PGA) is the first stable product, photosynthesis occurs in mesophyll cells, and photorespiration occurs while in $\mathrm{C}_{4}$ plants, the leaves show Kranz anatomy, phosphoenolpyruvate (PEP) is the first acceptor of $\mathrm{CO}_{2}$, oxaloacetate (OAA) is the first stable product, photosynthesis occurs in both mesophyll cells and bundle sheath cells, and photorespiration does not occur.

The chlorophyll content could depend on environmental and seasonal changes (Zhuang et al., 2020). Photosynthesis, stomatal conductance, transpiration and yield were higher but sub-stomatal $\mathrm{CO}_{2}$ concentration was lower under drought stress conditions than under control conditions in chickpea cultivars (Mafakheri et al., 2010).

\section{Chlorophyllide}

Chlorophyllide ais a metabolite late in the biosynthesis of bacteriochlorophylls and chlorophylls (Muller et al., 2011). Chlorophyllide $a$ is the prevailing form of chlorophyll in green algae and plants and the sole form of chlorophyll in many cyanobacteria; like most other chlorophylls, chlorophyll a includes of two moieties, the porphyrin macrocycle chlorophyllide $a$ and a branched-carbon phytyl tail (Vavilin and Vermaas, 2007). Chlorophyll $b$ is one of the main photosynthetic pigments of plants, and the regulation of chlorophyll $b$ biosynthesis is principal for plants in order to acclimate to changing environmental conditions, and in the chloroplast, chlorophyll $b$ is synthesized from chlorophyll $a$ by chlorophyllide $a$ oxygenase (CAO), a Riesketype monooxygenase (Sakuraba et al., 2009). Chlorophyll $b$ is considered as the antenna chlorophyll, and the binding of it by antenna proteins is influential for the correct assembly of the antenna complexes in thylakoid membranes (Voitsekhovskaja and Tyutereva, 2015).

\section{Protochlorophyllide oxidoreductase (POR)}

Protochlorophyllide (Pchlide) is a porphyrin dye, the main photosynthetic pigment, and one of the main intermediates in the biosynthetic pathway of chlorophyll (Chl) (Mysliwa-Kurdziel et al., 2013). The enzyme protochlorophyllide oxidoreductase ( $\mathrm{POR}$ ) catalyzes a principle light-driven reaction which triggers a profound transformation in plant development, in the chlorophyll biosynthetic pathway (Knaust et al., 1993; Armstrong et al., 2000; Heyes and Hunter, 2005).

As POR is activated by light, it can provide information on the way in which light energy can be harnessed to power enzyme reactions and it shows a unique opportunity to study catalysis at low temperatures and on ultrafast timescales which are not available for most analyses of enzyme function (Heyes and Hunter, 2005). POR finds in multiple isoforms that share high level of homology, and silencing of POR results in accumulation of protochlorophyllide (Pchlide) and complete loss of chlorophyllide (Chlide) (Talaat, 2013). The importance of carotenoid for membrane organization of NADPH POR was found (Denev et al., 2005). 
POR is a major enzyme for the light-induced greening of angiosperms (Reinbothe et al., 2003). Protochlorophyll (Pchl) and Protochlorophyllide (Pchlide) are naturally occurring prophyrins in plants; Pchl is more hydrophobic than Pchlide due to a long chain of phytol or its precursors attached to the tetrapyrrole ring (Mysliwa-Kurdziel et al., 2013). The nature of the solvent environment determined the excited-state relaxation of Pchlide (Dietzek et al., 2010). POR has a conserved Tyr and Lys residue in the enzyme active site like the other members of the short chain alcohol dehydrogenase/reductase family enzymes, which are involved in a proposed reaction mechanism involving proton transfer from the Tyr hydroxyl group to Pchlide (Menon et al., 2009). POR, together with DNA photolyase, is one of only the two enzymes that show a direct, natural requirement for light and because mixing techniques are no longer needed to initiate the reaction, it is possible to trigger catalysis at cryogenic temperatures and on very fast time scales (Mees et al., 2004); consequently, POR has proven to be an unique model system for studying the function of protein dynamics in driving enzyme catalysis (Heyes and Hunter, 2005).

It is reported that phytochrome A (phyA) down-regulates the synthesis of NADPH: protochlorophyllide (Pchlide) oxidoreductase and active Pchlide ${ }^{655}$ under far-red light (FR) (Sineshchekov et al., 2004). Dark-operative protochlorophyllide (Pchlide) oxidoreductase (DPOR) is a nitrogenase-like enzyme catalyzing a reduction of the $\mathrm{C} 17=\mathrm{C} 18$ double bond of Pchlide to form chlorophyllide a (Chlide) in bacteriochlorophyll biosynthesis, and DPOR contains of an ATP-dependent reducatse component, L-protein (a BchL dimer), and a catalytic constituent, NB-protein (a BchN-BchB heterotetramer), and the L-protein transfers electrons to the NB-protein to reduce Pchlide, which is associated with ATP hydrolysis (Nomata et al., 2016).

The NADPH-protochlorophyllide oxidoreductase (NPR) is a well-documented enzyme catalyzing photoconversion of protochlorophyllide to chlorophyllide $a$ and its expression is negatively adjusted by light (Kuroda et al., 1995). Oosawa et al. (2000) demonstrated that Arabidopsisthaliana are controlled by three POR isoforms, which are differentially controlled by development and light. Zhang et al. (2019) indicated that how the POR active site promotes light-driven reduction of protochlorophyllide by localized hydride transfer $\mathrm{NADPH}$ and long-range proton transfer along structurally defined proton-transfer pathways.

\section{Light-Dependent NADPH Protochlorophyllide Oxidoreductase (LPOR)}

The development of chloroplasts up to the stage of etioplasts which consist characteristic structures recognized as prolamellar bodies (PLBs) which have tubules joined together in a regular network and have a particular paracrystalline symmetry (Kowalewska et al., 2016). Protochlorophyllide (Pchlide) is a porphyrin dye and one of the main intermediates in the biosynthetic pathway of chlorophyll (Chl), the key photosynthetic pigment (Mysliwa-Kurdziel et al., 2013). Conversion of pchlide to chl includes two reactions, first is reduction of one double-bond in the porphyrin ring, leading to chlorophyllide (Chlide) formation, and the second is esterification of chlide by phytol or its unsaturated precursors (Willows, 2003; Eckhardt et al., 2004; Bollivar, 2006; Masuda, 2008; Masuda and Fujita, 2008). The enzyme protochlorophyllide oxidoreductase (POR) has a significant function in plant development, and it catalyzes one of the later steps in chlorophyll synthesis, the light-induced reduction of protochlorophyllide (PChlide) into chlorophyllide (Chlide) in the presence of NADPH (Garrone et al., 2015).

In angiosperms, Pchlide reduction to Chlide is completely light-dependent and catalyzed by a protochlorophyllide oxidoreductase (LPOR, EC 1.3.1.33), a photoenzyme (Mysliwa-Kurdziel et al., 2013), and angiosperms accumulate Pchlide in the dark but do not synthesize Chl (Kruk, 2005; Schoefs, 2005; Belyaeva and Litvin, 2007; Masuda, 2008; Reinbothe et al., 2010). In the absence of light, Pchlide accumulates in etioplasts, which develops instead of chloroplasts and may consist a regular paracrystalline lipid structure called as a prolamellar body (PLB) (Solymosi and Schoefs, 2008; Solymost and Schoef, 2010), and among different proteins recognized in PLB (Blomqvist et al., 2008), the most abundant is LPOR, which is found specially in the form of ternary Pchlide: LPOR: NADPH complexes (Ryberg and Sundqvist, 1982). 
LPOR is ubiquitous amongst eukaryotic phototrophs and is included of nucleus-encoded subunits that are post-translationally targeted to the chloroplasts (Aronsson et al., 2003); moreover, it is present in almost all chlorophyll manufacturing organisms but missing in photosynthetic bacteria (Adamson et al., 1997). LPOR belongs to the class of proteins known as short chain alcohol dehydrogenases and appears to have originally evolved in cyanobacteria because of strong evolutionary pressure and high oxygen sensitivity of DPOR (Yang and Cheng, 2004).

PLBs contain two spectral forms of the Pchlide-LPOR complexes with absorption maxima $\sim 640$ and 650 (Selstam et al., 2002). Schoefs and Frank (2004) concluded that only Pchlide, which forms ternary complexes with LPOR and NADPH (e.g. having the fluorescence maxima at 645 and $656 \mathrm{~nm}$ at $77 \mathrm{~K}$ ) can be reduced to Chlide by a millisecond flash illumination of saturating intensity. Short-wavelength forms were assigned to Pchlide which was unbound to the LPOR enzyme and mostly discovered in PTs (Ryberg and Sundqvist, 1982), and long-wavelength forms, found in PLBs, were attributed to aggregates of Pchlide: LPOR: NADPH complexes of different sizes that are stabilized by interaction of $\pi$ electrons of the neighboring pigment molecules (Boddi et al., 1989).

Two isoenzymes of the LPOR have been recognized: the LPOR-A which is accumulated in the dark and LPOR-B which is believed to work in plants under light exposure (Boddi et al., 1998). The redox state of NADPH in Pchlide: LPOR: NADPH complexes also have effect on PChlide spectral properties and may contribute to the heterogeneity (Frank et al., 1999). Mysliwa-Kurdziel et al. (2013) found the part of galactolipids of PLB in vivo in regulating the proportion between Pchlide aggregates and monomers, which is important for the proper assembly of ternary photoactive Pchlide: LPOR: NADPH complexes and for wellorganized PChlide to Chlide photoreduction.

The paracrystalline nature of PLBs is considered to be due to the aggregation of a complex containing protochlorophyllide (Pchlide), light-dependent protochlorophyllide oxidoreductase (LPOR), and NADPH (Pchlide-LPOR-NADPH) (Ryberg and Sundqvist, 1982). The role of the large pigment-protein complex Pchlide-LPOR in the formation of PLB membranes with a cubic phase structure is possibly because of the ability of this pigment-protein complex to form an oligomer and to anchor the LPOR protein into the membrane (Selstam, 1998). It has been reported that as a consequence of light-requirement of this enzyme, when plants are grown in darkness (etiolation), chlorophyll biosynthesis is terminated at the Pchlide phase and its accumulation is happened (Kruk et al., 2005). Park et al. (2002) observed that the proper composition of carotenoids in the PLB membrane can play an important function in the formation and maintenance of its paracrystalline structure. Grzyb et al. (2013) reported that AFM seems a promising method for scrutinizing PLB formation and the changes in PLB structure and it provides a notable chance to observe PLBs at a physiological temperature without the necessity fixation.

There is the evidence that heavy metals can interact with LPOR enzyme and inhibit Chl biosynthesis at the level of Pchlide to Chlide photoreduction (Stobart et al., 1985; Boddi et al., 1995; Berska et al., 2001). Mysliwa-Kurdziel and Strzalka (2005) concluded that the stability of PLB membranes may additionally be influenced because of the heavy metal treatment. Sperling et al. (1997) stated that highly regular structure of PLB membranes is thought to play a part in the protection of plants against photooxidation caused by intensive illumination of dark-adapted plants which is not appropriate for young plants.

LPORs were suggested to have evolved about 2 billion years ago in cyanobacteria as a consequence of increasing atmospheric oxygen levels (Yamazaki et al., 2006), and therefore, they are supposed to be absent in anoxygenic phototrophs (Kaschner et al., 2014). Kaschner et al. (2014) observed that in vitro and in vivo functional assays unequivocally prove light-dependent protochlorophyllide reduction by $D$. shibae DFL12 ${ }^{\mathrm{T}}$ and reveal LPORs are not restricted to cyanobacteria and plants. The three different isoforms of LPOR from A. thaliana were all proved to form Pchlide-LPOR-NADPH complexes with similar kinetic and spectral properties (Gabruk et al., 2015). Kaschner et al. (2014) also concluded that anoxygenic phototrophs only contain oxygen-sensitive dark-operative PORs (DPORs), which catalyze protochlorophyllide reduction independent of the presence of light, however, oxygenic phototrophs additionally contains oxygen-insensitive 
but light-dependent PORs (LPORs). Reduction of protochlorophyllide (Pchlide) to chlorophyllide (Chlide) by dark operative DPOR and light-dependent LPOR protochlorophyllide oxidoreductases is shown in Figure 1.

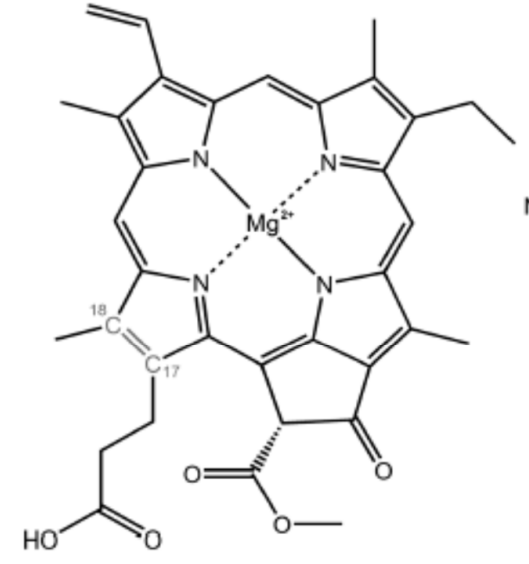

Protochlorophyllide

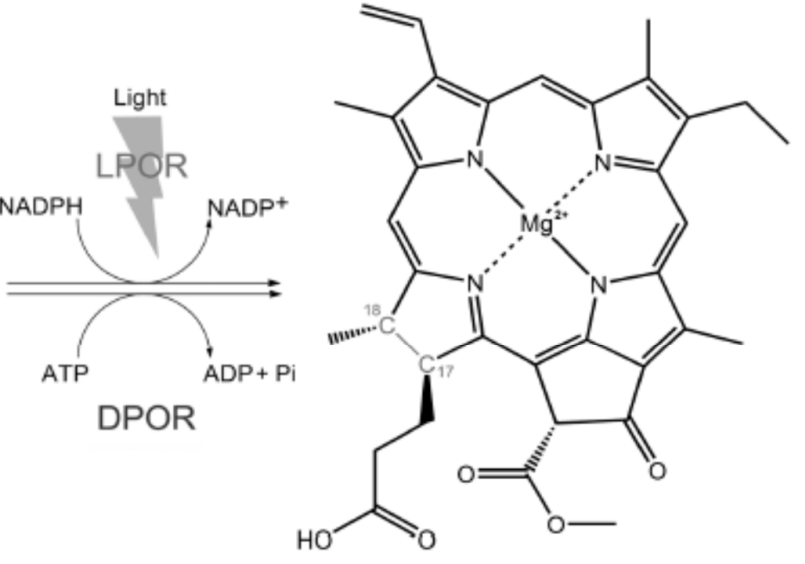

Chlorophyllide

Figure 1. Reduction of protochlorophyllide (Pchlide) to chlorophyllide (Chlide) by dark operative DPOR and light-dependent LPOR protochlorophyllide oxidoreductases

The $\mathrm{C} 17=\mathrm{C} 18$ double bond of Pchlide which is reduced by the respective protochlorophyllide reductase is shown in grey (Kaschner et al., 2014)

Ourteam worked together with the University of Manchester andShanghai Jiao Tong Hospital/Faculty of Basic Medicineand reportedcrystal structures of the apo-POR enzyme (Thermosynechococcus. elongatus, PDB code 6RNV), and NADPH-bound POR (Synechocystis and T. elongatus, PDB code 6G08 and 6RNW), solved at $1.3 \AA$, 1.9 $\AA$ and $1.9 \AA$ resolution, respectively (Zhang et al., 2019). This study reveals how the POR active site facilitates light-driven reduction of Pchlide by localized hydride transfer from NADPH and longrange proton transfer along structurally defined proton transfer pathways. Our study provides a structural basis for harnessing light energy to drive catalysis in this important chlorophyll biosynthetic enzyme, which is crucial for light-to-chemical energy conversion and unidirectional energy flow in the biosphere.

\section{Light-independent Pchlide Reductase (DPOR)}

In comparison with the dependence on light for the greening (Chl synthesis) of angiosperms, some photosynthetic organisms like non-flowering land plants, cyanobacteria, algae, and anoxygenic photosynthetic bacteria are capable of synthesizing Chls and BChls in the dark (Beigbeder et al., 1995; Nomata et al., 2005). These organisms have an unassociated Pchlide reductase enzyme called dark-operative Pchlide oxidoreductase (DPOR: light-independent Pchlide oxidoreductase). This enzyme catalyzes double-bond reduction in a lightindependent manner (Fujita, 1996; Armstrong et al., 1998; Fujita and Bauer, 2003), which has been wellknown as a mysterious enzyme because of the absence of a reliable assay system, which has hampered attempts at purification (Armstrong, 1998; Fujita and Bauer, 2003).

Nomata et al. (2006) reported that dark-operative protochlorophyllide reductase in bacteriochlorophyll biosynthesis is a nitrogenase-like enzyme including of L-protein (BchL-dimer) as a reductase component and NB-protein (BchN-BchB-heterotetramer) as a catalytic component. DPOR plays an important part in chlorophyll biosynthesis of gymnosperms, algae, mosses, ferns, and photosynthetic bacteria in the absence of 
light; although, DPOR shares notable amino acid sequence homologies with nitrogenase, only the initial catalytic steps look like nitrogenase catalysis (Brocker et al., 2008).

DPOR is made of electron donor $(\mathrm{BchL})$ and acceptor $(\mathrm{BchNB})$ component proteins; $\mathrm{BchNB}$ is further consisted of two subunits each of $B c h N$ and $B c h B$ arranged as an $\alpha_{2} \beta_{2}$ heterotetramer with two active sites for substrate reduction (Corless et al., 2020). Organisms containing DPOR can have functional chloroplasts in the dark and start photosynthesis upon exposure in light (Kusumi et al., 2006). A series of genetic researches that indicated three genes, $b c h L$, $b c h N$, and $b c h B$, encode subunits of DPOR in Rhodobacter capsulatus, Plectonema boryanum, and Chlamydomonas reinhardtii (Fujita, 1996; Suzuki et al., 1997; Fujita and Bauer, 2003; Maximova and Slovakova, 2014).

The DPOR subunits, BchL, BchN, and BchB, reveal significant sequence similarity to the nitrogenase subunits, NifH, NifD, and NifK, respectively, which exhibit that the mechanism of reducing the D-ring of Pchlide may be similar to the reduction of dinitrogen by nitrogenase (Fujita and Bauer, 2003). Two other DPOR subunits, BchN/ChlN, and BchB/ChlB show sequence similarity to both of NifD and NifK proteins, although at a lower level of 25-30\% similarity (including three Cys residues in NifD and one Cys residue in NifK) (Fujita, 1996; Fujita and Bauer, 2003).

DPOR includes of two separable components, L-protein (BchL), and NB-protein (BchN-BchB), and DPOR activity is dependent on ATP and a reducing agent such as dithionite, and L-protein was considered to function as an ATP-dependent electron donor to NB-protein, which provides the catalytic site for Pchlide reduction (Fujita and Bauer, 2000). L-protein and NB-protein of DPOR resemble Fe protein and MoFe protein of nitrogenase, respectively, in crystal structure (Sarma et al., 2008; Brocker et al., 2010; Muraki et al., 2010). The $[4 \mathrm{Fe}-4 \mathrm{~S}]$ cluster of the $\mathrm{L}$-protein is decreased by ferredoxin in vivo or dithionite in vitro (Fujita and Bauer, 2000; Nomata et al., 2005), and the electron is transferred to the NB-cluster in the NB-protein through a transient complex formation between the L-protein and the NB-protein (Nomata et al., 2013).

Takano et al. (2011) stated that the redox character of the NB cluster is responsible for why Asp36 is vital in DPOR, and also the unknown coordinating ligand of the [4Fe-4S] cluster in the D36A mutant of DPOR is a chloride ion. Nomata et al. (2008) showed that together with the Fe and sulfide contents, NBprotein carries two oxygen-tolerant [4Fe-4S] clusters. Kondo et al. (2011) suggested that Asp36 contributes to the low redox potential necessary to reduce protochlorophyllide. Yamamoto et al. (2009) suggested that the DPOR from an oxygenic photosynthetic organism did not obtain oxygen tolerance during evolution; but that the cyanobacterial cell developed a mechanism to protect DPOR from oxygen. Wu et al. (2001) found that a chlL-deletion mutant of Synerchocystis sp. PCC 6803 designated as chlL was inadequate to make significant amounts of chlorophyll in darkness, and PCR analysis confirmed that the chlL pseudorevertant mutant still lacked the chlL gene. Nazi and Khan (2013) found that the activation of dark-operative pathway requisites additional factors/genes to chlL and chlN genes to develop chlorophyll, and therefore photosynthetically competent chloroplasts.

Fujita et al. (1998) concluded that both LPOR and DPOR contribute to Chl synthesis in the cells growing in the light, the extent of the contribution by LPOR increases with increasing light intensity. Wei et al. (2004) also observed that at low light intensities, the Chl content in the mutant lacking DPOR was very low and its growth rate was retarded, but, under low light conditions, cyanobacteria might have the possibility to make efficient utilize of the small amount of light to boost photosynthetic efficiency for survival. Nomata et al. (2013) demonstrated that nicotinamide (NA) inhibits DPOR activity by blocking the electron transfer from L-protein to NB-protein, a reaction scheme of DPOR, in which the binding of protochlorophyllide (Pchlide) to the NB-protein precedes the electron transfer from the L-protein is proposed based on the NA impacts. Nomata et al. (2014) considered DPOR as a special iron-sulphur enzyme to form substrate radicals along with sequential proton- and electron-transfer steps with the protein folding very similar to that of nitrogenase.

The reaction of Pchlide reduction and inhibition of DPOR activity by NA, DPOR assay was carried out with purified L-protein and NB-protein in the absence (black line) or presence (500 $\mu \mathrm{M}$, red line) of NA are indicated in Figure 2. 

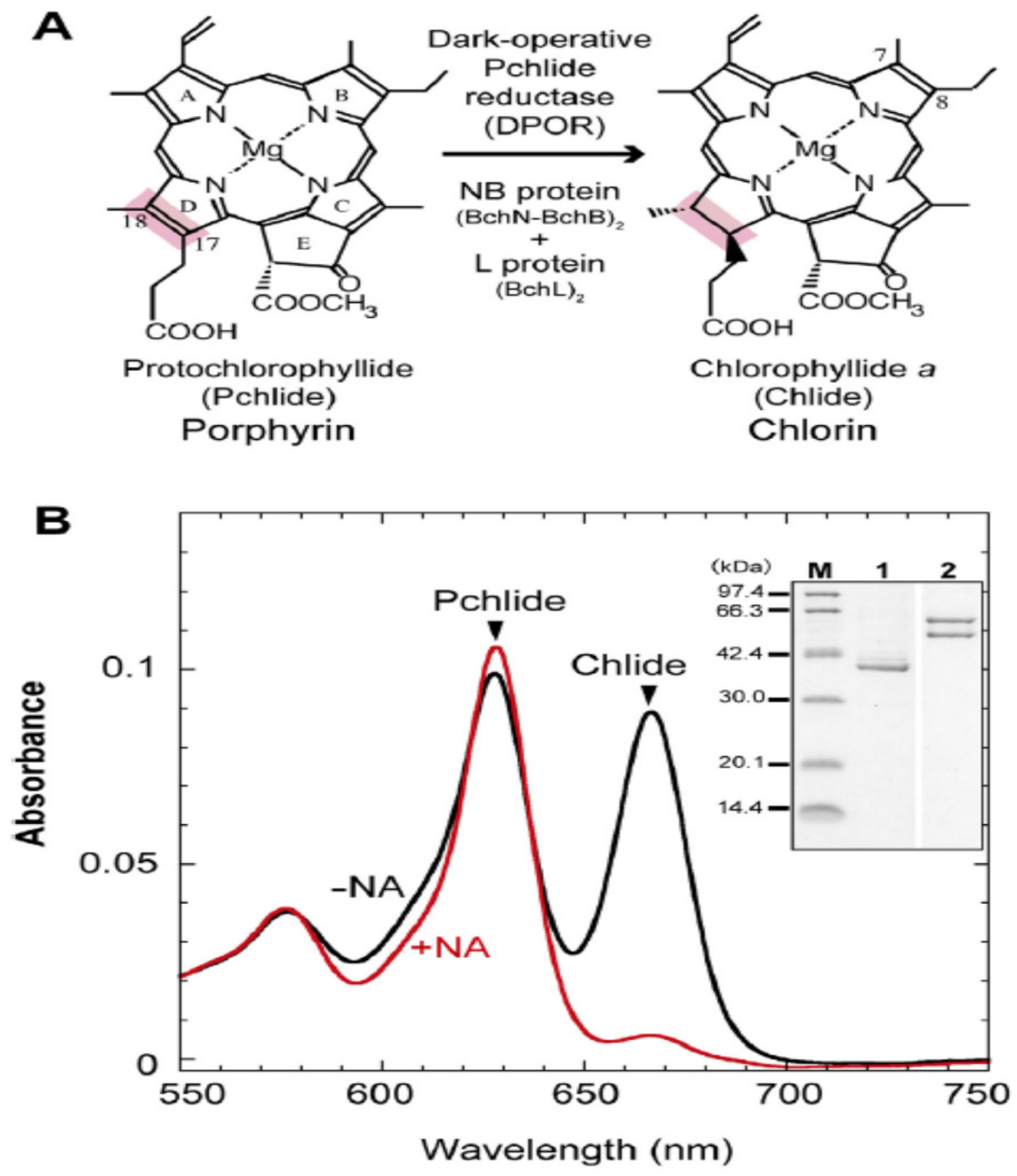

Figure 2. (A) The reaction of Pchlide reduction

The $\mathrm{C} 17=\mathrm{C} 18$ double bond of Pchlide is stereo specifically reduced by DPOR to form Chlide, the direct precursor of $\mathrm{Chl} \mathrm{a}$ in oxygenic photosynthetic organisms. In Bchl a biosynthesis Chlide is further reduced to 3-vinyl bacteriochlorophyllide a by Chlide oxidoreductase, another nitrogenase-like enzyme. (B) Inhibition of DPOR activity by NA, DPOR assay was carried out with purified L-protein and NB-protein in the absence (black line) or presence ( $500 \mu \mathrm{M}$, red line) of NA. The reactions were stopped by the addition of acetone and the absorption spectra were recorded. Inset. SDS-PAGE profile of the affinity purified L-protein (lane 1:1 $\mu \mathrm{g}$ ) and NB-protein (lane 2:2.5 $\mu \mathrm{g}$ ) (Reinbothe et al., 2010; Nomata et al., 2013).

Fujita and Bauer (2000) reported that ring D of pchlide can be reduced in the stereo-specific manner by two different enzymes, LPOR or DPOR, and the side chain of B-ring indicated by $\mathrm{R}$ is either vinyl or ethyl. Yamazaki et al. (2006) also showed that although these two enzymes carry out the same stereo-specific reduction of the double bond of the D-ring to produce chlorophyllide a (Chlide), the direct precursor for Chl a, they are structurally very dissimilar and use completely unalike mechanisms. So, DPOR and LPOR are analogous enzymes (Galperin et al., 1998). Shui et al. (2009) reported that transcripts levels of all DPOR genes are up-regulated approximately 2 -fold in green light $(\mathrm{GL})$ relative to levels in red light $(\mathrm{RL})$, whereas LPOR transcript levels are reduced in GL.

Yamamoto et al. (2014) found that both dark operative protochlorophyllide oxidoreductase (DPOR) and chlorophyllide a oxidoreductase (COR) include of two components, Fe protein and MoFe protein cognates, and COR catalyzes 8-vinyl reduction of 8-vinyl chlorophyllide a in addition to the known activity of 
$\mathrm{C} 7=\mathrm{C} 8$ double bond reduction. The sequential operation of DPOR and COR in the conversion of Pchlide to MV-Bchlide is presented in Figure 3. Two structurally unrelated Pchlide reductases are shown in Figure 4.

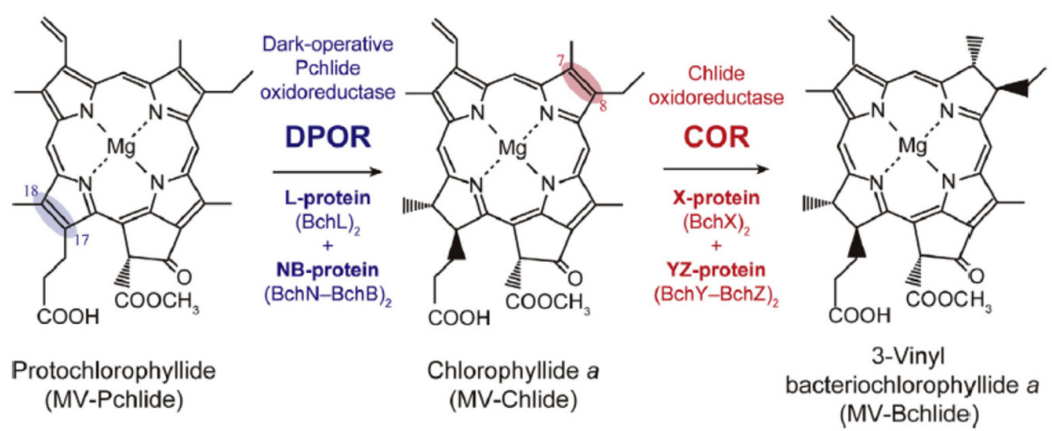

Figure 3. The sequential operation of DPOR and COR in the conversion of Pchlide to MV-Bchlide DPOR reduces the $\mathrm{C} 17=\mathrm{C} 18$ double bond (blue) of Pchlide to produce Chlide, and then COR reduces the $\mathrm{C} 7=\mathrm{C} 8$ double bond (red) of Chlide to produce MV-Bchlide (Yamamoto et al., 2014).

A

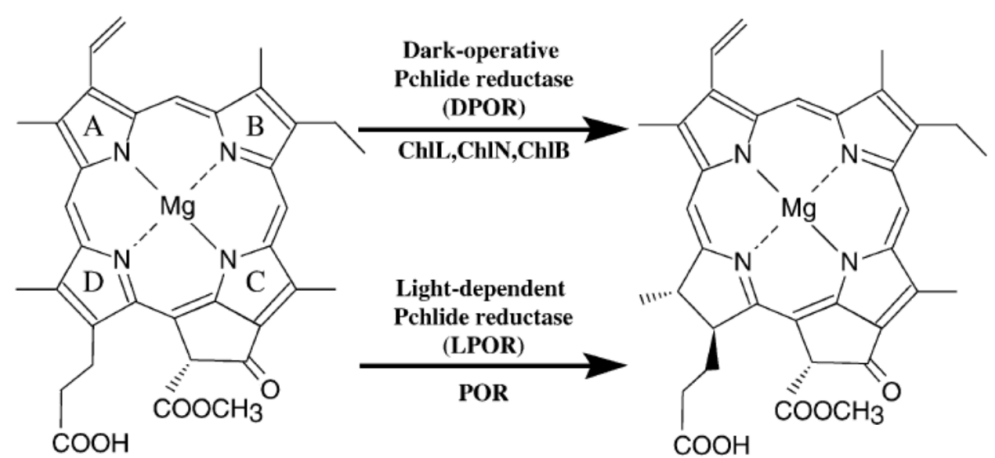

B

LPOR

DPOR

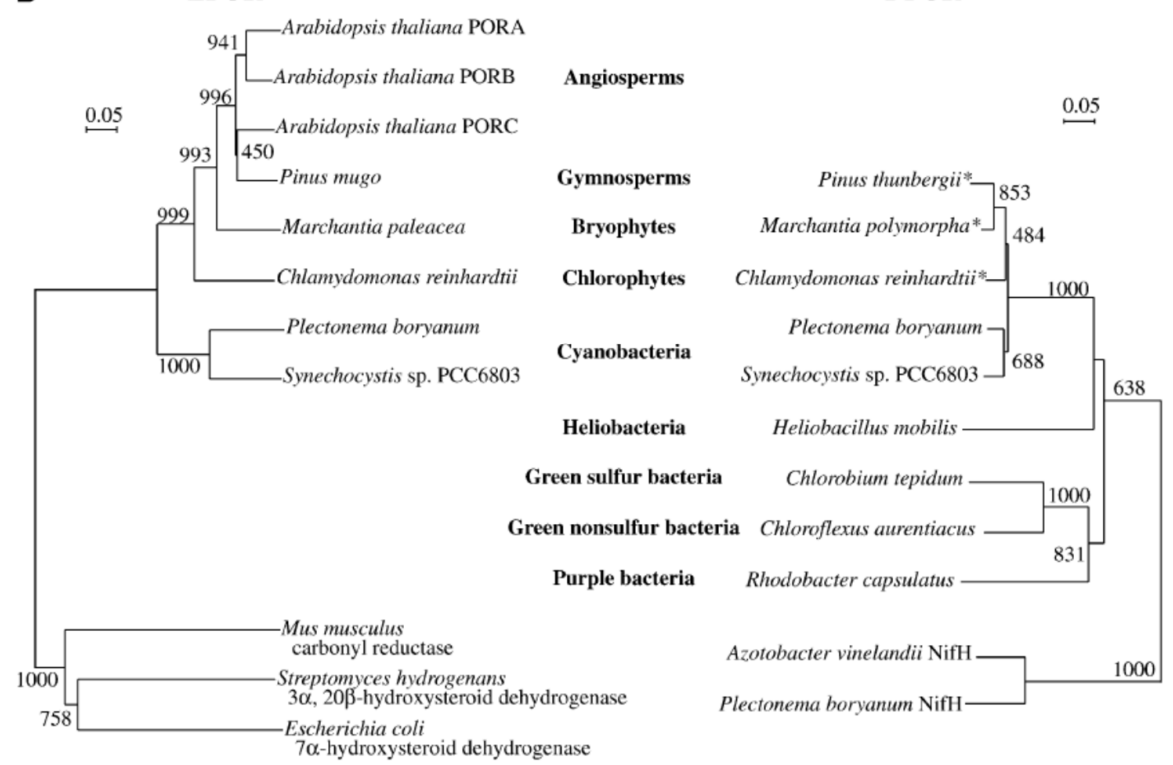

Figure 4. Two structurally unrelated Pchlide reductases

A. Pchlide reduction. The Pchlide D-ring is reduced by two different enzymes, DPOR and LPOR. B. Phylogenetic trees and distribution in extant photrophs of two Pchlide reductases. The amino acid sequences of LPOR and DPOR (Bchl/ChlL) were recovered from GenBank as illustrative for each main taxon of the extant phototrophs. Asterisks 
indicate that the genes are encoded in chloroplast genomes in these organisms. Each phylogenetic tree was constructed based on multiple sequence alignment by using ClustalX (version 1.81) and njplot. The three enzymes belonging to the SDR family and nitrogenase Fe-protein $(\mathrm{NifH})$ were used as outgroups for the LPOR and DPOR trees, respectively (Yamazaki et al., 2006).

\section{Conclusions}

Photosynthesis happens in mesophyll cell of the green leaves in a cell organelle called chloroplast, and within the chloroplast there is a membranous system including grana, the stroma lamellae and the fluid stroma. The membrane system traps the light energy and synthesizes ATP and NADPH, and this set of reaction which relies on light is called light reaction. In stroma, enzymatic reactions incorporate $\mathrm{CO}_{2}$ into the plant leading to the synthesis of sugar which in turn forms starch, and this set of reactions which are not directly dependent on light but are dependent on the products of light reactions, furthermore, ATP and NADPH is called dark reaction. Conversion of protochlorophyllide (Pchlide) into chlorophyllide (Chlide), a key step in chlorophyll biosynthesis, is mediated by a light-dependent NADPH: protochlorophyllide oxidoreducatse (POR). Two unrelated enzyme complexes have evolved to handle biosynthesis of chlorophyll, a light-dependent protochlorophyllide oxidoreductase (LPOR), and an ATP-dependent dark operative protochlorophyllide oxidoreductase (DPOR). POR has a conserved Tyr and Lys residue in the enzyme active site as the same as the other members of the short chain alcohol dehydrogenase/reductase family enzymes, which are active in a proposed reaction mechanism involving proton transfer from the Tyr hydroxyl group to Pchlide. Together with DNS photolyase, POR is one of the only two enzymes which show a natural, direct requirement for light. The enzyme POR has a significant function in plant development, and it catalyzes one of the later steps in chlorophyll synthesis, the light-induced reduction of PChlide into Chlide in the presence of NADPH. DPOR plays an important role in chlorophyll biosynthesis of gymnosperms, ferns, algae, mosses, and photosynthetic bacteria in the absence of light, although, DPOR shares considerable amino acid sequence homologies with nitrogenase, only the initial catalytic steps look like nitrogenase catalysis. DPOR is made of electron donor (BchL) and acceptor (BchNB) component proteins; $\mathrm{BchNB}$ is further consisted of two subunits each of BchN and $B c h B$ arranged as an $\alpha_{2} \beta_{2}$ heterotetramer with two active sites for substrate reduction. The DPOR subunits, $\mathrm{BchL}, \mathrm{BchN}$, and $\mathrm{BchB}$, reveal significant sequence similarity to the nitrogenase subunits, $\mathrm{NifH}, \mathrm{NifD}$, and NifK, respectively, which exhibit that the mechanism of reducing the D-ring of Pchlide may be similar to the reduction of dinitrogen by nitrogenase. DPOR has been considered as a special iron-sulphur enzyme to form substrate radicals along with sequential proton- and electron-transfer steps with the protein folding very similar to that of nitrogenase. The study of the structure of LPOR has implications for deep understanding of lightto-chemical energy conversion in the biosphere and ultimately, for the design of new chemical / biological photocatalysts. Perhaps in near future scientists will be able to apply existing LPOR and DPOR structural data to design new nitrogen-fixing enzymes that can express and function in eukaryotic cells.

\section{Authors' Contributions}

All authors read and approved the final manuscript.

\section{Acknowledgements}

This work was supported by the National Key R\&D Program of China (Research grant 2019YFA0904700). This research was also funded by the Natural Science Foundation of Beijing, China (Grant No. M21026). 


\section{Conflict of Interests}

The authors declare that there are no conflicts of interest related to this article.

\section{References}

Adamson HY, Hiller RG, Walmsley J (1997). Protochlorophyllide reduction and greening in angiosperms: an evolutionary perspective. Journal of Photochemistry and Photobiology B: Biology 41:201-221. https://doi.org/10.1016/s1011-1344(97)00105-x

Armstrong GA (1998). Greening in the dark: light-independent chlorophyll biosynthesis from anoxygenic photosynthetic bacteria to gymnosperms. Journal of Photochemistry and Photobiology B: Biology 43:87-100. https://doi.org/10.1016/s1011-1344(98)00063-3

Armstrong GA, Apel K, Rudiger W (2000). Does a light-harvesting protochlorophyllide $a / b$-binding protein complex exist? Trends in Plant Science 5(1):40-44. https://doiorg/10.1016/s1360-1385(99)01513-7

Aronsson H, Sundqvist C, Dahlin C (2003). POR hits the road: import and assembly of a plastid protein. Plant Molecular Biology 51:1-7. https://doi.org/10.1023/a:1020795415631

Beigbeder A, Vavadakis M, Navakoudis E, Kotzabasis K (1995). Influence of polyamine inhibitors on light-independent and light-dependent chlorophyll biosynthesis and on the photosynthetic rate. Journal of Photochemistry and Photobiology B: Biology 28:235-242. https://doi.org/10.1016/1011-1344(95)07113-g

Belyaeva OB, Litvin FF (2007). Photoactive pigment-enzyme complexes of chlorophyll precursor in plant leaves. Biochemistry (Moscow) 72:1458-1477. https://doi.org/10.1134/s0006297907130044

Berska J, Mysliwa-Kurdziel B, Strzalka K (2001). Transformation of protochlorophyllide to chlorophyllide in wheat under heavy metal stress. In: Proceedings of the 12th International Congress on Photosynthesis, CSIRO, pp. S2015 .

Bjorn LO, Papageorgiou GC, Blankenship RE, Govindjee (2009). A viewpoint: why chlorophyll a? Photosynthesis Research 99:85-98. https://doi.org/10.1007/s11120-008-9395-x

Blomqvist LA, Ryberg M, Sundqvist C (2008). Proteomic analysis of highly purified prolamellar bodies reveals their significance in chloroplast development. Photosynthesis Research 96:37-50. https://doi.org/10.1007/s11120$007-9281-y$

Boddi B, Lindsten A, Ryberg M, Sundqvist C (1989). On the aggregational states of protochlorophyllide and its protein complexes in wheat etioplasts. Physiologia Plantarum 76:135-143. https://doi.org/10.1111/j.13993054.1989.tb05622.x

Boddi B, Oravecz AR, Lehoczki E (1995). Effect of cadmium on organization and photoreduction of protochlorophyllide in dark grown leaves and etioplast inner membrane preparations of wheat. Photosynthetica 31:411-420.

Boddi B, Kis-Petik K, Kaposi AD, Fidy J, Sundqvist C (1998). The two spectroscopically different short wavelength protochlorophyllide forms in pea epicotyls are both monomeric. Biochimica et Biophysica Acta 1365:531-540. https://doi.org/10.1016/s0005-2728(98)00106-6

Bollivar DW (2006). Recent advances in chlorophyll biosynthesis. Photosynthesis Research 90:173-194. https://doi.org/10.1007/s11120-006-9076-6

Brocker MJ, Watzlich D, Uliczka F, Virus S, Saggu M, Lendzian F, Scheer H, Rudiger W, Moser J, Jahn D (2008). Substrate recognition of nitrogenase-like dark operative protochlorophyllide oxidoreductase from Prochlorococcusmarinus. The Journal of Biological Chemistry 283(44):2987329881. https://doi.org/10.1074/jbc.m805206200

Brocker MJ, Schomburg S, Heinz DW, Jahn D, Schubert WD, Moser J (2010). Crystal structure of the nitrogenase-like dark operative protochlorophyllide oxidoreductase catalytic complex (ChlN/ChlB). Journal of Biological Chemistry 285:27336-27345. https://doi.org/10.1074/jbc.m110.126698

Chen M (2014). Chlorophyll modifications and their spectral extension in oxygenic photosynthesis. Annual Review of Biochemistry 83:317-340. https://doi.org/10.1146/annurev-biochem-072711-162943

Corless EI, Bennett B, Antony E (2020). Substrate recognition induces sequential electron transfer across subunits in the nitrogenase-like DPOR complex. Journal of Biological Chemistry 295(39):13630-13639. https://doi.org/10.1074/jbc.ra120.015151 
Denev ID, Yahubyan GT, Minkov IN, Sundqvist C (2005). Organization of protochlorophyllide oxidoreductase in prolamellar bodies isolated from etiolated carotenoid-deficient wheat leaves as revealed by fluorescence probes. Biochimica et Biophysica Acta 1716:97-103. https://doi.org/10.1016/j.bbamem.2005.09.001

Dietzek B, Tschierlei S, Hanf R, Seidel S, Yartsev A, Schmitt M, Hermann G, Popp J (2010). Dynamics of charge separation in the excited-state chemistry of protochlorophyllide. Chemical Physics Letters 492(1-3):157-163. https://doi.org/10.1016/j.cplett.2010.04.027

Eckhardt U, Grimm B, Hortensteiner S (2004). Recent advances in chlorophyll biosynthesis and breakdown in higher plants. Plant Molecular Biology 56:1-14. https://doi.org/10.1007/s11103-004-2331-3

Frank F, Bereza B, Boddi B (1999). Protochlorophyllide-NADP ${ }^{+}$and protochlorophyllide-NADPH complexes and their regeneration after flash illumination in leaves and etioplast membranes dark-grown wheat. Photosynthesis Research 59:53-61.

Frankenberg C, Berry J (2018). Solar induced chlorophyll fluorescence: origins, relation to photosynthesis and retrieval. Comprehensive Remote Sensing 3:143-162. https://doi.org/10.1016/b978-0-12-409548-9.10632-3

Fujita Y (1996). Protochlorophyllide reduction: a key step in the greening of plants. Plant and Cell Physiology 37:411421. https://doi.org/10.1093/oxfordjournals.pcp.a028962

Fujita Y, Takagi H, Hase T (1998). Cloning of the gene encoding a protochlorophyllide reductase: the physiological significance of the co-existence of light-dependent and -independent protochlorophyllide reduction systems in the Cyanobacterium Plectonema boryanum. Plant and Cell Physiology 39(2):177-185. https://doi.org/10.1093/oxfordjournals.pcp.a029355

Fujita Y, Bauer CE (2000). Reconstitution of light-independent protochlorophyllide reductase from purified BchL and $\mathrm{BchN}-\mathrm{BchB}$ subunits. In vitro confirmation of nitrogenase-like features of a bacteriochlorophyll biosynthetic enzyme. Journal of Biological Chemistry 275:23583-23588. https://doi.org/10.1074/jbc.m002904200

Fujita Y, Bauer CE (2003). The light-independent protochlorophyllide reductase: a nitrogenase-like enzyme catalyzing a key reaction for greening in the dark. In: Kadish KM, Smith KM, Guilard R (Eds). Porphyrin Handbook, Chlorophylls and Bilins: Bionsynthesis, Synthesis and Degradation. Vol. 13, Academic Press, New York, pp 109156. https://doi.org/10.1016/b978-0-08-092387-1.50010-2

Gabruk M, Stecka A, Strzalka W, Kruk J, Strzalka K, Mysliwa-Kurdziel B (2015). Photoactive protochlorophyllideenzyme complexes reconstituted with PORA, PORB, and PORC proteins of $A$. thaliana: Fluorescence and catalytic properties. Plos One 10(2):e0116990. https://doi.org/10.1371/journal.pone.0116990

Galperin MY, Walker DR, Koonin EV (1998). Analogous enzymes: independent inventions in enzyme evolution. Genome Research 8:779-790. https://doi.org/10.1101/gr.8.8.779

GarroneA, Archipowa N, Zipfel PF, Hermann G, Dietzek B (2015). Plant protochlorophyllide oxidoreductases A and B - Catalytic efficiency and initial reaction steps. Journal of Biological Chemistry. https://doi.org/10.1074/jbc.m115.663161

Gotoh E, Kobayashi Y, Tsuyama M (2010). The post-illumination chlorophyll fluorescence transient indicates the RuBP regeneration limitation of photosynthesis in low light in Arabidopsis. FEBS Letters 584(14):3061-3064. https://doi.org/10.1016/j.febslet.2010.05.039

Grajek H, Rydzynski D, Piotrowicz-Cieslak A, Herman A, Maciejczyk M, Wieczorek Z (2020). Cadmium ionchlorophyll interaction-examination of spectral properties and structure of the cadmium-chlorophyll complex and their relevance to photosynthesis inhibition. Chemosphere 261:127434. https://doi.org/10.1016/j.chemosphere.2020.127434

Green BR (2011). After the primary endosymbiosis: an update on the chromalveolate hypothesis and the origins of algae with Chl c. Photosynthesis Research 107:103-115. https://doi.org/10.1007/s11120-010-9584-2

Grzyb JM, Solymosi K, Strzalka K, Mysliwa-Kurdziel B (2013). Visualization and characterization of prolamellar bodies with atomic force microscopy. Journal of Plant Physiology 170:1217-1227. https://doi.org/10.1016/j.jplph.2013.04.017

Heyes DJ, Hunter CN (2005). Making light work of enzyme catalysis: protochlorophyllide oxidoreductase. Trends in Biochemical Sciences 30(11):642-649. https://doi.org/10.1016/j.tibs.2005.09.001

Humphrey AM (1980). Chlorophyll. Food Chemistry 5(1):57-67. https://doi.org/10.1016/0308-8146(80)90064-3

Hunter CN, Artymiuk PJ, van Amerongen H (1994). Photosynthesis: Many chlorophylls make light work. Current Biology 4(4):344-346. https://doi.org/10.1016/s0960-9822(00)00075-0 
Kaschner M, Loeschcke A, Krause J, Minh BQ, Heck A, Endres S, ... Krauss U (2014). Discovery of the first lightdependent protochlorophyllide oxidoreductase in anoxygenic phototrophic bacteria. Molecular Microbiology 93(5):1066-1078. https://doi.org/10.1111/mmi.12719

Knaust R, Seyfried B, Schmidt L, Schulz R, Senger H (1993). Phototransformation of monovinyl and divinyl protochlorophyllide by NADPH: Protochlorophyllide oxidoreductase of barley expressed in Escherichia coli. Journal of Photochemistry and Photobiology B: Biology 20(2-3):161-166. https://doi.org/10.1016/10111344(93)80146-Z

Kondo T, Nomata J, Fujita Y, Itoh S (2011). EPR study of 1Asp-3Cys ligated 4Fe-4S iron-sulfur cluster in NB-protein (BchN-BchB) of a dark-operative protochlorophyllide reductase complex. FEBS Letters 585:214-218. https://doi.org/10.1016/j.febslet.2010.11.044

Kowalewska L, Mazur R, Suski S, Garstka M, Mostowska A (2016). Three-dimensional visualization of the tubularlamellar transformation of the internal plastid membrane network during runner bean chloroplast biogenesis. The Plant Cell 28:875-891.https://doi.org/10.1105/tpc.15.01053

Kruk J (2005). Occurrence of chlorophyll precursors in leaves of cabbage heads- the case of natural etiolation. Journal of Photochemistry and Photobiology B: Biology 80:187-194. https://doi.org/10.1016/j.jphotobiol.2005.04.003

Kuroda H, Masuda T, Ohta H, Shioi Y, Takamiya K (1995). Light-enhanced gene expression of NADPHprotochlorophyllide oxidoreductase in cucumber. Biochemical and Biophysical Research Communications 210(2):310-316. https://doi.org/10.1006/bbrc.1995.1662

Kusumi J, Sato A, Tachida H (2006). Relaxation of function constraints on light-independent protochlorophyllide oxidoreductase in Thuja. Molecular Biology and Evolution 23:941-948. https://doi.org/10.1093/molbev/msj097

Li Y, Song H, Zhou L, Xu Z, Zhou G (2019). Vertical distributions of chlorophyll and nitrogen and their associations with photosynthesis under drought and re-watering regimes in a maize field. Agricultural and Forest Meteorology 272-273:40-54. https://doi.org/10.1016/j.agrformet.2019.03.026

Liu X, Li L, Zhang B, Zing L, Li L (2020). AhHDA1-mediated AhGLK1 promoted chlorophyll synthesis and photosynthesis regulates recovery growth of peanut leaves after water stress. Plant Science 294:110461. https://doi.org/10.1016/j.plantsci.2020.110461

Lu X, Liu Z, Zhao F, Tang J (2020). Comparison of total emitted solar-induced chlorophyll fluorescence (SIF) and topof-canopy (TOC) SIF in estimating photosynthesis. Remote Sensing of Environment 251:112083. https://doi.org/10.1016/j.rse.2020.112083

Mafakheri A, Siosemardeh A, Bahramnejad B, Struik PC, Sohrabi Y (2010). Effect of drought stress on yield, proline and chlorophyll contents in three chickpea cultivars. Australian Journal of Crop Science 4(8):580-585.

Mandal R, Dutta G (2020). From photosynthesis to biosensing: Chlorophyll proves to be a versatile molecule. Sensors International 1:100058. https://doi.org/10.1016/j.sintl.2020.100058

Masuda T (2008). Recent overview of the Mg branch of the tetrapyrrole biosynthesis leading to chlorophylls. Photosynthesis Research 96:121-143. https://doi.org/10.1007/s11120-008-9291-4

Masuda T, Fujita Y (2008). Regulation and evolution of chlorophyll biosynthesis. Photochemical and Photobiological Sciences 7:1131-1149. https://doi.org/10.1039/b807210h

Maximova N, Slovakova L (2014). Accumulation of photosynthetic pigments in Larix deciduas Mill. and Picea abies (L.) Karst. Cotyledons treated with 5-aminolevulinic acid under different irradiation. Photosynthetica 52(2):203-210. https://doi.org/10.1007/s11099-014-0019-8

Mees A, Klar T, GnauP, Hennecke U, Eker APM, Carell T, Essen L-O (2004). Crystal structure of a photolyase bound to a CPD-like DNA lesion after in situ repair. Science 306:1789-1793.https://doi.org/10.1126/science.1101598

Menon BRK, Waltho JP, Scrutton NS, Heyes DJ (2009). Cryogenic and laser photoexcitation studies identify multiple roles for active site residues in the light-driven enzyme protochlorophyllide oxidoreductase. The Journal of Biological Chemistry 284(27):18160-18166. https://doi.org/10.1074/jbc.m109.020719

Muller AH, Gough SP, Bollivar DW, Meldal M, Willows RD, Hansson M (2011). Methods for the preparation of chlorophyllide a: An intermediate of the chlorophyll biosynthetic pathway. Analytical Biochemistry 419(2):271276. https://doi.org/10.1016/j.ab.2011.08.028

Muraki N, Nomata J, Ebata K, Mizoguchi T, Shiba T, Tamiaki H, Kurisu G, Fujita Y (2010). X-ray crystal structure of the light-independent protochlorophyllide reductase. Nature 465:110-114. https://doi.org/10.1038/nature08950 
Mysliwa-Kurdziel B, Strzalka K (2005). Influence of Cd (II), Cr (VI), and Fe (III) on early steps of deetiolation process in wheat: fluorescence spectral changes of protochlorophyllide and newly formed chlorophyllide. Agriculture, Ecosystems and Environment 106:199-207. https://doi.org/10.1016/jagee.2004.10.008

Mysliwa-Kurdziel B, Kruk J, Strazalka K (2013). Protochlorophyllide in model systems- An approach to in vivo conditions. Biophysical Chemistry 175-176:28-38. https://doi.org/10.1016/j.bpc.2013.02.002

Nazir S, Khan MS (2013). Integration of novel chlorophyll genes from black pine into the chloroplast genome of tobacco. Pakistan Journal of Botany 45(S1):595-600. https://doi.org/10.1007/s11033-012-1953-9

Nick S, Meurer J, Soll J, Ankele R (2013). Nucleus-encoded light-harvesting chlorophyll a/b proteins are imported normally into chlorophyll b-free chloroplasts of Arabidopsis. Molecular Plant 6:860-871. https://doi.org/10.1093/mp/sss 113

Nomata J, Swem LR, Bauer CE, Fujita Y (2005). Over-expression and characterization of dark-operative protochlorophyllide reductase from Rhodobactercapsulatus. Biochimica et Biophysica Acta 1708:229-237. https://doi.org/10.1016/j.bbabio.2005.02.002

Nomata J, Kitashima M, Inoue K, Fujita Y (2006). Nitrogenase Fe protein-like Fe-S cluster is conserved in L-protein (BchL) of dark-operative protochlorophyllide reductase from Rhodobacter capsulatus. FEBS Letters 580:61516154. https://doi.org/10.1016/j.febslet.2006.10.014

Nomata J, Ogawa T, Kitashima M, Inoue K, Fujita Y (2008). NB-protein (BchN-BchB) of dark-operative protochlorophyllide reductase is the catalytic component containing oxygen-tolerant Fe-S clusters. FEBS Letters 582:1346-1350. https://doi.org/10.1016/j.febslet.2008.03.018

Nomata J, Kondo T, Itoh S, Fujita Y (2013). Nicotinamide is a specific inhibitor of dark-operative protochlorophyllide oxidoreductase, a nitrogenase-like enzyme, from Rhodobacter capsulatus. FEBS Letters 587:3142-3147. https://doi.org/10.1016/j.febslet.2013.07.054

Nomata J, Kondo T, Mizoguchi T, Tamiaki H, Itoh S, Fujita Y (2014). Dark-operative protochlorophyllide oxidoreductase generates substrate radicals by an iron-sulphur cluster in bacteriochlorophyll biosynthesis. Scientific Reports. 4:5455. https://doi.org/10.1038/srep05455

NomataJ, Terauchi K, Fujita Y (2016). Stoichiometry of ATP hydrolysis and chlorophyllide formation of fark-operative protochlorophyllide oxidoreductase from Rhodobacter capsulatus. Biochemical and Biophysical Research Communications 470(3):704-709. https://doi.org/10.1016/j.bbrc.2016.01.070

Oosawa N, Masuda T, Awai K, Fusada N, Shimada H, Ohta H, Takamiya K-I (2000). Identification and light-induced expression of a novel gene of NADPH-protochlorophyllide oxidoreductase isoform in Arabidopsis thaliana. FEBS Letters 474:133-136. https://doi.org/10.1016/s0014-5793(00)01568-4

Park H, Kreunen SS, Cuttriss AJ, Della Penna D, Pogson BJ (2002). Identification of the carotenoid isomerase provides insight into carotenoid biosynthesis, prolamellar body formation and photomorphogenesis. Plant Cell 14:321332. https://doi.org/10.1105/tpc.010302

Reinbothe C, Buhr F, Pollmann S, Reinbothe S (2003). In vitro reconstitution of light-harvesting PORprotochlorophyllide complex with protochlorophyllides $a$ and $b$. The Journal of Biological Chemistry 278(2):807815. https://doi.org/10.1074/jbc.m209738200

Reinbothe C, El Bakkouri M, Buhr F, Muraki N, Nomata J, Kurisu G, Fujita J, Reinbothe S (2010). Chlorophyll biosynthesis: spotlight on protochlorophyllide reduction. Trends in Plant Science 15:614-624. https://doi.org/10.1016/j.tplants.2010.07.002

Ryberg M, Sundqvist C (1982). Characterization of prolamellar bodies and prothylakoids fractionated from wheat etioplasts. Physiologia Plantarum 56:125-132. https://doi.org/10.1111/j.1399-3054.1982.tb00313.x

Sakuraba Y, Tanaka R, Yamasato A, Tanaka A (2009). Determination of a chloroplast degron in the regulatory domain of chlorophyllide $a$ oxygenase. The Journal of Biological Chemistry 284(52):3668936699. https://doi.org/10.1074/jbc.m109.008144

Sarma R, Barney B, Hamilton T, Jones A, Seefeldt L, Peters J (2008). Crystal structure of the L protein of Rhodobacter sphaeroides light-independent protochlorophyllide reductase with MgADP bound: a homologue of the nitrogenase Fe protein. Biochemistry 47:13004-13015.https://doi.org/10.2210/pdb3end/pdb

Schoefs B, Franck F (2004). Protochlorophyllide reduction: mechanisms and evolution. Photochemistry and Photobiology 78:543-557. https://doi.org/10.1562/0031-8655(2003)0780543prmae2.0.co2

Schoefs B (2005). Protochlorophyllide reduction- what is new in 2005? Photosynthetica 43:329343. https://doi.org/10.1007/s11099-005-0056-4 
Selstam E (1998). Development of thylakoid membranes with respect to lipids. In: Lipids in Photosynthesis: Structure, Function and Genetics. Paul-Andre S and Norio M (Eds). The Netherlands: Springer, pp. 209-224. https://doi.org/10.1007/0-306-48087-5_11

Selstam E, Schelin J, Brain T, Williams WP (2002). The effects of low pH on the properties of protochlorophyllide oxidoreductase and the organization of prolamellar bodies of maize (Zea mays). European Journal of Biochemistry 269:2336-2346. https://doi.org/10.1046/j.1432-1033.2002.02897.x

Shahrajabian MH, Sun W, Cheng Q (2021) The importance of flavonoids and phytochemicals of medicinal plants with antiviral activities. Mini Review in Organic Chemistry. 18. https://doi.org/10.2174/1570178618666210707161025

Shui J, Saunders E, Needleman R, Nappi M, Cooper J, Hall L, Kehoe D, Stowe-Evans E (2009). Light-dependent and light-independent protochlorophyllide oxidoreductases in the chromatically adapting Cynanobacterium fremyella diplosiphon UTEX 481. Plant and Cell Physiology 50(8):1507-1521. https://doi.org/10.1093/pcp/pcp095

Sineshchekov V, Belyaeva O, Sudnitsin A (2004). Up-regulation by phytochrome A of the active protochlorophyllide, Pchlide ${ }^{655}$, biosynthesis in dicots under far-red light. Journal of Photochemistry and Photobiology B: Biology 74(1):47-54. https://doi.org/10.1016/j.jphotobiol.2004.02.001

Soleymani A, Shahrajabian MH, Khoshkharam M (2016). The impact of barley residue management and tillage on forage maize. Romanian Agricultural Research 33:161-167.

Solymosi K, Schoefs B (2008). Prolamellar body: a unique plastid compartment, which does not only occur in dark-grown leaves. In: Schoefs B (Ed). Plant Cell Compartments- Selected Topics, Res. Sign Post, India, pp 152-202.

Solymosi K, Schoefs B (2010). Etioplast and etio-chloroplast formation under natural conditions: the dark side of chlorophyll biosynthesis in angiosperms. Photosynthesis Research 105:143166. https://doi.org/10.1007/s11120-010-9568-2

Sperling U, van Cleve B, Frick G, Apel K, Armstrong GA (1997). Over-expression of light-dependent PORA and PORB in plants depleted of endogeneous POR by far-red enhances seedling survival in white light and protects against photooxidative damage. The Plant Journal 12:649-658. https://doi.org/10.1046/j.1365-313x.1997.d01-11.x

Sun W, Shahrajabian MH, Cheng Q (2019). The insight and survey on medicinal properties and nutritive components of shallot. Journal of Medicinal Plant Research 13(18):452-457. https://doi.org/10.5897/jmpr2019.6836

Sun W, Shahrajabian MH, Cheng Q (2021). Fenugreek cultivation with emphasis on historical aspects and its uses in traditional medicine and modern pharmaceutical science. Mini Reviews in Medicinal Chemistry 21(6):724-730. https://doi.org/10.2174/1389557520666201127104907

Suzuki JY, Bollivar DW, Bauer CE (1997). Genetic analysis of chlorophyll biosynthesis. Annual Review of Genetics 31:61-89. https://doi.org/10.1146/annurev.genet.31.1.61

Takano Y, Yonezawa Y, Fujita Y, Kurisu G, Nakamura H (2011). Electronic structures of a [4Fe-4S] cluster, $\left[\mathrm{Fe}_{4} \mathrm{~S}_{4}\left(\mathrm{SCH}_{3}\right)_{3}\left(\mathrm{CH}_{3} \mathrm{COO}\right)\right]$, in dark-operative protochlorophyllide oxidoreductase (DPOR). Chemical Physics Letters 503:296-300. https://doi.org/10.1016/j.cplett.2011.01.026

Talaat NB (2013). RNAi based simultaneous silencing of all forms of light-dependent NADPH: protochlorophyllide oxidoreductase genes result in the accumulation of protochlorophyllide in tobacco (Nicotiana tabacum). Plant Physiology and Biochemistry 71:31-36. https://doi.org/10.1016/j.plaphy.2013.06.025

Van der Tol C, Vehoef W, Rosema A (2009). A model for chlorophyll fluorescence and photosynthesis at leaf scale. Agricultural and Forest Meteorology 149(1):96-105. https://doi.org/10.1016/j.agrformet.2008.07.007

Vavilin D, Vermaas W (2007). Continuous chlorophyll degradation accompanied by chlorophyllide and phytol reutilization for chlorophyll synthesis in Synechocystis sp. PCC. 6830. Biochimica et Biophysica Acta 1767:920929. https://doi.org/10.1016/j.bbabio.2007.03.010

Voitsekhovskaja OV, Tyutereva EV (2015). Chlorophyll $b$ in angiosperms: Functions in photosynthesis, signaling and ontogenetic regulation. Journal of Plant Physiology 189:51-64. https://doi.org/10.1016/j.jplph.2015.09.013

Wei H, Qingyu W, Jiujiu Y (2004). Contribution of DPOR at low light intensity to chlorophyll biosynthesis and growth in the Synechocystissp. PCC 6803. Tsinghua Science and Technology 9(1):69-75.

Willows RD (2003). Biosynthesis of chlorophylls from protoporphyrin IX. Natural Product Reports 20:327-341. https://doi.org/10.1039/b110549n

Wu Q, Yu J, Zhao N (2001) Partial recovery of light-independent chlorophyll biosynthesis in the chlL-deletion mutant of Synechocystis sp. PCC 6803. IUBMB Life 51:289-293. https://doi.org/10.1080/152165401317190789 
Yamamoto H, Kurumiya S, Ohashi R, Fujita Y (2009). Oxygen sensitivity of a nitrogenase-like protochlorophyllide reductase from the cyanobacterium Leptolyngbya boryana. Plant and Cell Physiology 50(9):1663-1673. https://doi.org/10.1093/pcp/pcp111

Yamamoto H, Kato M, Yamanashi K, Fujita Y (2014). Reconstitution of a sequential reaction of two nitrogenase-like enzymes in the bacteriochlorophyll biosynthetic pathway of Rhodobacter capsulatus. Biochemical and Biophysical Research Communications 448:200-205. https://doi.org/10.1016/j.bbrc.2014.04.087

Yamazaki S, Nomata J, Fujita Y (2006). Differential operation of dual protochlorophyllide reductases for chlorophyll biosynthesis in response to environmental oxygen levels in the cyanobacterium Leptolyngbya boryana. Plant Physiology 142:911-922. https://doi.org/10.1104/pp.106.086090

Yang J, Cheng Q (2004). Origin and evolution of the light-dependent protochlorophyllide oxidoreductase (LPOR) genes. Plant Biology (Stuttgart, Germany) 6(5):537-544. https://doi.org/10.1055/s-2004-821270

Zhang S, Heyes DJ, Feng L, Sun W, Johannissen LO, Liu H, ... Scrutton NS (2019) Structural basis for enzymatic photocatalysis in chlorophyll biosynthesis. Nature 574(7780):722-725. https://doi.org/10.1038/s41586-019$1685-2$

Zhuang J, Wang Y, Chi Y, Zhou L, Chen J, Zhou W, Song J, Zhao N, Ding J (2020). Drought stress strengthens the link between chlorophyll fluorescence parameters and photosynthetic traits. Peer Journal 8:e10046. https://doi.org/10.7717/peerj.10046
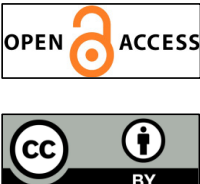

The journal offers free, immediate, and unrestricted access to peer-reviewed research and scholarly work. Users are allowed to read, download, copy, distribute, print, search, or link to the full texts of the articles, or use them for any other lawful purpose, without asking prior permission from the publisher or the author.

License - Articles published in Notulae Botanicae Horti Agrobotanici Cluj-Napoca are Open-Access, distributed under the terms and conditions of the Creative Commons Attribution (CC BY 4.0) License. (c) Articles by the authors; UASVM, Cluj-Napoca, Romania. The journal allows the author(s) to hold the copyright/to retain publishing rights without restriction. 